\title{
Lipid and antioxidant changes in spermatozoa and seminal plasma throughout the reproductive period of bulls
}

\author{
K. A. Kelso, A. Redpath*, R. C. Noble and B. K. Speake \\ Department of Biochemical Sciences, Scottish Agricultural College, Auchincruive, Ayr KA6 5HW, UK
}

\begin{abstract}
The lipid compositions and associated antioxidant capacities of spermatozoa and seminal plasma from bulls were examined at the beginning, middle and end of their reproductive period. The reduction in concentration and motility of spermatozoa associated with ageing was accompanied by a large decrease in lipid concentrations within the seminal plasma; this change in lipid concentration was accompanied by an increase in the proportion of phospholipid. By contrast, the proportion of phospholipids in the spermatozoa was significantly reduced. The major phospholipid fractions within both the spermatozoa and seminal plasma were phosphatidyl choline and phosphatidyl ethanolamine. With increasing age there was a large decrease in the proportion of phosphatidyl ethanolamine and a commensurate increase in that of phosphatidyl choline within the spermatozoa and seminal plasma. These major changes in phospholipids were accompanied by a decrease in the amount of phosphatidyl inositol and an incr sse in that of cardiolipin in both spermatozoa and seminal plasma. The reductions in the proportions of phosphatidyl ethanolamine were accompanied by extensive reductions in the content of the major polyunsaturated fatty acids, arachidonic 20:4 (n-6) and docosahexaenoic 22:6 (n-3); there was a decrease also in the concentration of 22:6 (n-3) in phosphatidyl choline. The changes in lipid composition owing to ageing were associated with a marked reduction within the seminal plasma of the major antioxidant enzyme systems, glutathione peroxidase and superoxide dismutase.
\end{abstract}

\section{Introduction}

Since the nineteenth century researchers have reported that lipids are a basic component of semen, contributing to the membrane structure of spermatozoa, the metabolism of the sperm cells and to their ability to capacitate and fertilize the female gamete (Mann and Lutwak-Mann, 1981). The fatty acid composition of the spermatozoa and seminal plasma from many animal species has been reported and a key finding was the presence of extremely high concentrations of polyunsaturated phospholipids (Pickett and Komarek, 1966; Neill and Masters, 1972; Poulos and White, 1973; Poulos et al., 1973; Scott, 1973; Lin et al., 1993). In most mammals the substantial concentration of polyunsaturates present is characteristically dominated by docosahexaenoic acid (22:6 n-3), (Neill and Masters, 1972; Poulos et al., 1973; Evans and Setchell, 1978; Nissen and Kreysel, 1983; Lin et al., 1993). In bulls in particular, docosahexaenoic acid accounts for around $55-60 \%$ of the total phospholipid fatty acids, with especially high concentrations, $40 \%$ and $80 \%$, occurring within the phosphatidyl ethanolamine and phosphatidyl choline fractions, respectively (Poulos et al., 1973; Jain and Anand, 1976). This contrasts significantly with domestic avian species in which docosatetraenoic acid (22:4 $\mathrm{n}-6)$ predominates (Ravie and Lake, 1985; Kelso et al., 1996).

*Present address: Scottish Livestock Services (SLS), Newlands Cattle Breeding Centre, Scone, Perth PH2 6NN, UK.

Revised manuscript received 24 July 1996.
There is considerable evidence to indicate that the lipid composition of the sperm membrane is a major determinant of the cold sensitivity, motility and overall viability of spermatozoa (Bakst and Sexton, 1979; Elias et al., 1979; Bearer and Friend, 1982; Hammerstedt, 1993; Roldan and Harrison, 1993). The presence of such high concentrations of long chain polyunsaturated fatty acids within the lipid structure of sperm cells requires efficient antioxidant systems to defend against peroxidative damage and associated sperm dysfunction (Jones et al., 1979; Wishart, 1984; Alvarez and Storey, 1989; Selley et al., 1991; Cecil and Bakst, 1993; Aitken, 1994). Thus the maintenance of high fertility appears to require the presence of optimal concentrations of polyunsaturated fatty acids together with associated antioxidant capacity.

The present report concerns the investigation of the relationship in bulls between changes in some major indicators of semen quality during ageing and some major lipid metabolic parameters; these include the nature of the lipids, their fatty acids and the amounts of antioxidant enzymes in both the cellular and plasma components of the semen.

\section{Materials and Methods}

\section{Bulls and semen collection}

Samples were obtained from three groups each comprising four male Holstein/Friesian bulls used for semen collection and 
artificial insemination at Scottish Livestock Services, Perth. At the beginning of the experiment the bulls were $2-3$ years old in the young group, 5-6 years old in the intermediate group and $>9$ years old in the older group. The bulls were housed in single pens in a controlled environment during the months of October to May. Dietary treatments were identical, each animal receiving $3 \mathrm{~kg}$ twice per day of a standard commercial diet with $16 \%$ crude protein and $12.5 \mathrm{MJ} \mathrm{kg}^{-1}$ of metabolizable energy. Regular lipid analysis of feed was undertaken to establish the lipid and fatty acid composition. Silage was fed ad libitum and was of average to good quality with dry matter $270.8 \mathrm{~g} \mathrm{~kg}^{-1}$. Semen samples were collected routinely from all bulls by artificial vagina after I h of 'teasing' (Hafez, 1993) approximately once every 2 weeks and once a week throughout the experimental collection period.

\section{Evaluation of spermatozoa}

Within 20 min of collection, measurements were made of major semen parameters, including ejaculate volume using a graduated tube, sperm concentration using an IMV photometer (Instruments Medicines Veterinaire, L'Aigle) and subjective motility on a scale of $0-5$ (Logue and Greg, 1987). A portion of fresh semen was also used for the immediate assay of superoxide dismutase and glutathione peroxidase.

\section{Lipid extraction and analysis}

Semen was measured and diluted with an equal volume of $0.85 \%(\mathrm{w} / \mathrm{v})$ sodium chloride solution and centrifuged at $700 \mathrm{~g}$ for $20 \mathrm{~min}$ at $4^{\circ} \mathrm{C}$. The upper diluted plasma layer was transferred to a fresh test tube, the wash procedure repeated with $2 \mathrm{ml}$ of $0.85 \%(\mathrm{w} / \mathrm{v})$ sodium chloride and the final cell pellet was resuspended in $2 \mathrm{ml} 0.85 \%(\mathrm{w} / \mathrm{v})$ sodium chloride

Total lipid was extracted from the spermatozoa and seminal plasma preparations after homogenization in a suitable excess of chloroform:methanol $(2: 1 \mathrm{v}: \mathrm{v})$; this has been established as a highly efficient solvent mixture to ensure the quantitative and reproducible extraction of lipid (Christie, 1982). The lipids were fractionated into their major classes (phospholipid, free cholesterol, triacylglycerol, free fatty acids and cholesterol ester) by thin layer chromatography (TLC) on silica gel G using a solvent system of hexane:diethyl ether:formic acid (80:20:1 $\mathrm{v}: \mathrm{v}: \mathrm{v})$. After visualization under UV light after spraying with $0.1 \%(w: v)$ 2,7-dichlorofluorescein in methanol, the separated bands were scraped from the plates. Phospholipid was eluted from the silica by washing three times with $2 \mathrm{ml}$ methanol, and the other lipid classes were similarly eluted with diethyl ether. The esterified lipid fractions were subjected to transmethylation by refluxing with methanol:toluene:sulphuric acid (20:10:1, v:v:v) in the presence of a pentadecanoic acid standard (Christie et al., 1970). The resultant fatty acid methyl esters were analysed by a $I \mu l$ injection via a CP9010 autosampler (Chrompack, London) onto a $30 \mathrm{~m} \times 0.25 \mathrm{~mm}$ diameter, $0.25 \mu \mathrm{m}$ film thickness Carbowax capillary column (Econo-Cap, Alltech UK Ltd, Carnforth) fitted to a Chrompack CP9001 instrument (Chrompack). Integration of the peaks using an 'EZ-Chrom' Data Handling System (Speck Analytical, Alloa) enabled the determination of the fatty acid composition
(\% w/w of total fatty acids). The amount of each lipid class was calculated by comparison of the total fatty acid peak areas with that of the pentadecanoic fatty acid standard (Christie et al., 1970). Free cholesterol was determined by standard colorimetric assay (Boehringer, Lewes).

Individual phospholipid classes were separated by high performance thin layer chromatography (HPTLC) using a solvent system of methyl acetate:isopropanol:chloroform: methanol:0.25\% ( $w / v$ in $\left.\mathrm{H}_{2} \mathrm{O}\right) \mathrm{KCl}(25: 25: 25: 10: 9 \mathrm{v}: \mathrm{v})$, as described by Olsen and Henderson (1989). After charring (Kelso et al., 1996) quantification was performed by densitometry using a Shimadzu CS-9001 PC dual wavelength flying spot thin layer scanner (Dyson Instruments, Houghton Le Spring). Separation of phosphatidyl choline and phosphatidyl ethanolamine from sperm cells before fatty acid analysis, as outlined above, was performed by high performance liquid chromatography (HPLC) according to the method described by Christie (1988).

\section{Enzyme quantification}

All assays were performed on a portion of seminal plasma from each bull using standard kit systems (Randox Limited, Crumlin). The glutathione peroxidase (GPX) assay was based on the enzyme-catalysed oxidation of reduced glutathione by cumene hydroperoxide coupled to the reduction of the oxidized glutathione by NADPH. Units of GPX activity are expressed as $\mu$ mol NADPH oxidized $\min ^{-1}$. Quantification of the superoxide dismutase (SOD) activity used xanthine and xanthine oxidase to generate superoxide radicals followed by their detection via formazan dye formation. Units of SOD activity are defined as the amount of SOD required to inhibit the rate of formazan dye formation by $50 \%$ under the conditions specified by the kit manufacturers.

\section{Statistical analysis}

Student's $t$ test was used for statistical comparison of intermediate and old aged bulls to the values obtained for the young group of bulls. The data encompassed four samples per group for lipid analysis, eight (two ejaculates per bull) from each group for SOD and GPX and all associated quantifications described, that is, sperm concentration and motility.

\section{Results}

The concentration of spermatozoa in the semen samples from the intermediate and old bulls was significantly less than that observed for the younger age group and was accompanied by a significant reduction in the motility of the sperm cells (Table 1). There was a marked decrease in the activities of both glutathione peroxidase and superoxide dismutase of the seminal plasma with increasing age.

Total lipid concentrations in the seminal plasma showed a wide variation (Table 2). Phospholipid was by far the predominant lipid fraction within the spermatozoa and was accompanied by substantial proportions of free cholesterol. The concentration of phospholipid in the seminal plasma was considerably lower than for the spermatozoa, with higher 
Table 1. Semen characteristics for young, intermediate and old bulls

\begin{tabular}{lccc}
\hline & \multicolumn{3}{c}{ Age of bulls } \\
\cline { 2 - 4 } Characteristic & Young & Intermediate & Old \\
\hline Sperm concentration $\left(10^{9}\right.$ cells ml $^{-1}$ ) & $1.38 \pm 0.08$ & $1.00 \pm 0.10^{b}$ & $0.89 \pm 0.12^{b}$ \\
Motility (0-5), subjective & 5 & 4 & 2 \\
Glutathione peroxidase (units ml & & $10.7 \pm 2.1^{\mathrm{c}}$ & $5.4 \pm 2.5^{\mathrm{b}}$ \\
Superoxide dismutase (units $\mathrm{ml}^{-1}$ seminal plasma) & $17.6 \pm 1.5$ & $3.5 \pm 0.4$ & $3.3 \pm 0.2^{\mathrm{c}}$ \\
\hline
\end{tabular}

Values are means \pm SEM of four observations from each age group.

Significance of difference compared with values for young bulls: ${ }^{b} P<0.01,{ }^{c} P<0.05$. Comparisons without a superscript were not significant.

Table 2. Lipid composition of spermatozoa and seminal plasma from young, intermediate and older bulls

\begin{tabular}{|c|c|c|c|}
\hline \multirow[b]{2}{*}{ Lipid composition } & \multicolumn{3}{|c|}{ Age of bulls } \\
\hline & Young & Intermediate & Old \\
\hline \multicolumn{4}{|l|}{ Spermatozoa } \\
\hline Total lipid ( $\mu$ g per $10^{9}$ cells) & $595.5 \pm 42.5$ & $654.3 \pm 29.9$ & $670.9 \pm 99.2$ \\
\hline \multicolumn{4}{|l|}{ Lipid class (\% w/w of total lipid) } \\
\hline Phospholipid & $74.3 \pm 1.3$ & $74.8 \pm 2.2$ & $62.8 \pm 1.8^{b}$ \\
\hline Free cholesterol & $16.7 \pm 0.8$ & $21.8 \pm 0.2^{b}$ & $19.6 \pm 1.7$ \\
\hline Triacylglycerol & $2.4 \pm 0.4$ & $2.2 \pm 0.5$ & $4.5 \pm 1.0$ \\
\hline Free fatty acid & $2.8 \pm 0.6$ & $2.8 \pm 0.3$ & $5.9 \pm 3.0$ \\
\hline Cholesterol ester & $3.1 \pm 0.2$ & $2.9 \pm 0.3$ & $3.2 \pm 0.5$ \\
\hline \multicolumn{4}{|l|}{ Seminal plasma } \\
\hline Total lipid ( $\mu \mathrm{g} \mathrm{ml}^{-1}$ seminal plasma) & $570.0 \pm 63.2$ & $333.3 \pm 48.9^{c}$ & $215.3 \pm 45.0^{\mathrm{b}}$ \\
\hline \multicolumn{4}{|l|}{ Lipid class (\% w/w of total lipid) } \\
\hline Phospholipid & $39.2 \pm 3.8$ & $44.2 \pm 1.2$ & $47.0 \pm 0.5$ \\
\hline Free cholesterol & $35.3 \pm 0.6$ & $34.5 \pm 0.8$ & $36.2 \pm 2.0$ \\
\hline Triacylglycerol & $6.4 \pm 0.6$ & $5.9 \pm 1.4$ & $5.6 \pm 0.7$ \\
\hline Free fatty acid & $9.4 \pm 1.8$ & $7.0 \pm 0.9$ & $4.7 \pm 1.2$ \\
\hline Cholesterol ester & $8.0 \pm 2.2$ & $7.2 \pm 0.1$ & $5.3 \pm 1.7$ \\
\hline
\end{tabular}

Values are means \pm SEM of four observations from each age group.

Significance of difference compared with values for young bulls. ${ }^{b} P<0.01,{ }^{c} P<0.05$. Comparisons without a superscript were not significant.

concentrations of cholesterol ester, triacylglycerol and free fatty acid being present. Although maintaining an overall similarity in lipid composition to the young donors, the spermatozoa from the older bulls showed significant reductions in the phospholipid and free cholesterol concentrations. There was no difference in the lipid composition of the seminal plasma between the young, intermediate and old donors.

Within the spermatozoa of the young donors, by far the major phospholipid fractions were phosphatidyl ethanolamine and phosphatidyl choline, accompanied by smaller proportions of cardiolipin and sphingomyelin (Table 3). In the older donors, the spermatozoa showed a considerable reduction in the proportions of the phosphatidyl ethanolamine and a concomitant increase in the proportions of phosphatidyl choline. Phosphatidyl choline was by far the major lipid fraction within the seminal plasma of the young donors. The pattern of change displayed with age by the seminal plasma was similar to that displayed by the spermatozoa, namely a reduction in the proportion of phosphatidyl ethanolamine and an increase in that of phosphatidyl choline. Whereas cardiolipin was present only to a very minor extent within the seminal plasma of the young bulls, it accounted for a larger proportion of the total phospholipid in the older bulls within both the sperm cells and seminal plasma.

The major carriers of the polyunsaturated fatty acids within the spermatozoa in all three groups of bulls were the phosphatidyl ethanolamine and phosphatidyl choline fractions. The major polyunsaturates in the phosphatidyl ethanolamine were arachidonic acid (20:4n-6) and docosahexaenoic acid (22:6n-3), while in phosphatidyl choline, docosahexaenoic acid (22:6 n-3) predominated (Table 4). Together, these two acids accounted for $62 \%$ of the total fatty acid present within the phosphatidyl ethanolamine fraction of the young bulls compared with a total concentration of over $90 \%$ in the phosphatidyl choline fraction. The phosphatidyl choline fraction was characterized by the domination of docosahexaenoic acid compared with all other 
Table 3. Phospholipid classes of spermatozoa and seminal plasma from young, intermediate and old bulls

\begin{tabular}{|c|c|c|c|}
\hline \multirow[b]{2}{*}{ Phospholipid classes } & \multicolumn{3}{|c|}{ Age of bulls } \\
\hline & Young & Intermediate & Old \\
\hline \multicolumn{4}{|l|}{ Spermatozoa } \\
\hline \multicolumn{4}{|c|}{ Phospholipid fraction ( $\% \mathrm{w} / \mathrm{w}$ of total phospholipid) } \\
\hline Phosphatidyl choline & $41.9 \pm 1.3$ & $48.9 \pm 1.8^{c}$ & $57.0 \pm 0.4^{\mathrm{a}}$ \\
\hline Phosphatidyl ethanolamine & $28.6 \pm 1.6$ & $28.3 \pm 0.7$ & $21.0 \pm 1.2^{c}$ \\
\hline Phosphatidyl serine & $<1.0$ & $<1.0$ & $<1.0$ \\
\hline Phosphatidyl inositol & $4.3 \pm 0.5$ & $2.3 \pm 0.5^{c}$ & $2.0 \pm 0.5^{b}$ \\
\hline Sphingomyelin & $10.2 \pm 1.3$ & $13.7 \pm 0.8$ & $6.6 \pm 3.3$ \\
\hline Cardiolipin & $8.9 \pm 0.9$ & $6.9 \pm 0.3$ & $11.6 \pm 0.4^{\circ}$ \\
\hline \multicolumn{4}{|l|}{ Seminal plasma } \\
\hline \multicolumn{4}{|c|}{ Phospholipid fraction ( $\% \mathrm{w} / \mathrm{w}$ of total phospholipid) } \\
\hline Phosphatidyl choline & $44.2 \pm 1.3$ & $53.8 \pm 0.4^{b}$ & $51.3 \pm 1.6^{\circ}$ \\
\hline Phosphatidyl ethanolamine & $28.7 \pm 1.0$ & $26.3 \pm 0.7$ & $22.5 \pm 0.7^{\mathrm{b}}$ \\
\hline Phosphatidyl serine & $6.3 \pm 0.8$ & $6.3 \pm 1.4$ & $8.6 \pm 1.1$ \\
\hline Phosphatidyl inositol & $3.0 \pm 0.8$ & $2.3 \pm 0.5$ & $1.5 \pm 0.3$ \\
\hline Sphingomyelin & $15.3 \pm 1.9$ & $13.7 \pm 1.9$ & $15.1 \pm 0.5$ \\
\hline Cardiolipin & $<1.0$ & $<1.0$ & $1.3 \pm 0.6^{\mathrm{a}}$ \\
\hline
\end{tabular}

Values are means \pm SEM of four observations from each age group.

Significance of difference compared with values for young bulls: ${ }^{a} P<0.001,{ }^{b} P<0.01,{ }^{c} P<0.05$. Comparisons without a superscript were not significant.

Table 4. Fatty acid composition of the phosphatidyl ethanolamine and phosphatidyl choline fractions of spermatozoa phospholipids from young, intermediate and old bulls

\begin{tabular}{lccc}
\hline & \multicolumn{3}{c}{ Age of bulls } \\
\cline { 2 - 4 } Fatty acid composition & Young & Intermediate & Old \\
\hline Phosphatidyl ethanolamine & & & \\
Fatty acid (\% w/w of total fatty acid) & & & $13.8 \pm 0.5^{\mathrm{c}}$ \\
$16: 0$ & $8.6 \pm 1.2$ & $16.5 \pm 2.9^{\mathrm{c}}$ & $12.9 \pm 0.9$ \\
$18: 0$ & $14.8 \pm 1.5$ & $13.9 \pm 1.6$ & $10.9 \pm 1.5^{\mathrm{c}}$ \\
$18: 1(\mathrm{n}-9)$ & $4.3 \pm 0.1$ & $9.5 \pm 1.7^{\mathrm{c}}$ & $3.7 \pm 0.3$ \\
$18: 1(\mathrm{n}-7)$ & $3.4 \pm 1.2$ & $<1.0$ & $3.1 \pm 0.1^{\mathrm{a}}$ \\
$20: 3(\mathrm{n}-3)$ & $\mathrm{nd}$ & $\mathrm{nd}$ & $9.9 \pm 1.0^{\mathrm{b}}$ \\
$20: 4(4-6)$ & $15.0 \pm 0.4$ & $10.0 \pm 0.8^{\mathrm{a}}$ & $30.9 \pm 1.7^{\mathrm{c}}$ \\
$22: 6(\mathrm{n}-3)$ & $47.3 \pm 4.6$ & $39.2 \pm 2.4$ & \\
Phosphatidyl choline & & & $4.8 \pm 0.4$ \\
Fatty acid (\% w/w of total fatty acid) & $5.5 \pm 0.4$ & $8.4 \pm 1.0^{\mathrm{c}}$ & $3.4 \pm 1.4$ \\
$16: 0$ & $1.8 \pm 0.7$ & $3.7 \pm 0.8$ & $1.3 \pm 0.2$ \\
$18: 0$ & $<1.0$ & $5.2 \pm 1.3^{\mathrm{a}}$ & $1.3 \pm 0.1$ \\
$18: 1(\mathrm{n}-9)$ & $1.2 \pm 0.1$ & $1.3 \pm 0.1$ & $76.0 \pm 3.8$ \\
$22: 5(\mathrm{n}-3)$ & $91.1 \pm 1.2$ & $76.0 \pm 3.8^{\mathrm{b}}$ & \\
$22: 6(\mathrm{n}-3)$ & & & \\
\hline
\end{tabular}

Values are means \pm SEM of four observations from each age group. nd $=$ not detectable.

Significance of difference compared with values for young bulls: ${ }^{a} P<0.001,{ }^{b} P<0.01,{ }^{C} P<0.05$. Comparisons without a superscript were not significant.

fatty acids. The major difference between the three groups of bulls was the very large reduction that occurred in the concentrations of both arachidonic and docosahexaenoic acids within the phosphatidyl ethanolamine fraction of the spermatozoa with age, and in the docosahexaenoic acid of the phosphatidyl choline fraction. Thus the concentrations of arachidonic acid and docosahexaenoic acid in the phosphatidyl ethanolamine fraction of the spermatozoa from the old bulls were reduced by approximately $30 \%$ and $35 \%$, respectively, in comparison with young bulls. The phosphatidyl choline fraction of the spermatozoa exhibited a decrease of approximately $15 \%$ in docosahexaenoic acid (22:6n-3) with age. 


\section{Discussion}

In general the composition and specific lipid and fatty acid features of the spermatozoa and seminal plasma displayed by the young bulls in this study were similar to those reported previously for sexually active bulls (Neill and Masters, 1972; Poulos et al., 1973; Scott, 1973). As with other animal species, the lipids of the spermatozoa and, to a large extent those of the seminal plasma, in bulls were dominated by polyunsaturated phospholipids, comprising mainly phosphatidyl ethanolamine and phosphatidyl choline. In common with other mammals, but in contrast to domestic avian species in which docosatetraenoic acid, 22:4 (n-6) predominates (Darin-Bennett et al., 1974; Howarth et al., 1977; Ravie and Lake, 1985; Kelso et al., 1996), both spermatozoa and seminal plasma in bulls were characterized by the presence of very high concentrations of $\mathrm{C} 20$ and C22 polyunsaturates, but most notably docosahexaenoic acid (Neill and Masters, 1972; Poulos et al., 1973; Darin-Bennett et al., 1974; Nissen and Kreysel, 1983; Lin et al., 1993).

A feature of ageing and associated changes in semen quality in the present study was the considerable reduction in overall lipid content in the seminal plasma. In the absence of a glycolytic substrate, both endogenous and exogenous lipids play a substantial role in the supply of energy for the motility and viability of spermatozoa (Scott and Dawson, 1968; Scott, 1973). In contrast to the present observations, it has been shown that a characteristic of decreased fertility in humans is a higher concentration of lipid within both the cellular and plasma components of the semen (Sebastian et al., 1987). This effect highlights the differences in sperm metabolism between the two species and during ageing in the bull; high concentrations of exogenous fructose are known to be present in bull semen (Garner and Hafez, 1993) and in conjunction with reduced antioxidant protection by glutath ione peroxidase and superoxide dismutase systems, an adverse effect on glycolytic function may result in the utilization of exogenous lipids for metabolic function of spermatozoa (Mann et al., 1980b, cited by Mann and Lutwak-Mann, 1981; Griveau et al., 1995).

The major feature of the present investigations was the highly significant reduction with ageing in the phosphatidyl ethanolamine fraction within spermatozoa and its replacement by phosphatidyl choline, and the specific reduction in the content of arachidonic and docosahexaenoic acids. In addition, there was an associated alteration in the proportion of other phospholipid classes in both the cellular and seminal plasma components. Functional roles of the lipids in overall fertility, motility and freezing capabilities (Darin-Bennett et al., 1974; Nissen et al., 1981; Nissen and Kreysel, 1983), in particular in association with their highly polyunsaturated fatty acid content, may be related to general effects on the biophysical properties of the membranes, such as fluidity and permeability (Hammerstedt, 1993). Lipids also have an important role to play in membrane fusion (Bearer and Friend, 1982), leukotriene production (Oliw and Sprecher, 1989) and signal transduction (Roldan and Harrison, 1993), which have all been implicated in the acrosome reaction and fertilization process. From the results of this study, reductions in the concentrations of the long chain polyunsaturated fatty acids, specifically $22: 6(\mathrm{n}-3)$, and antioxidant protection may account for the decrease in semen quality parameters and ultimate fertilizing ability of spermatozoa from ageing bulls.

The causes of the lipid compositional changes observed between the three groups of bulls can only be speculated upon. The bulls were maintained on identical diets throughout the duration of the investigation and therefore differences arising from dietary and environmental provisions are unlikely. One possibility is a significant reduction in testicular enzyme capacity for the synthesis of $\mathrm{C} 20$ and $\mathrm{C} 22$ polyunsaturates or their subsequent incorporation into the phospholipid fractions during differentiation of the spermatozoa; $\Delta 6$ desaturase activity is known to decrease with age (Brenner, 1989). In addition, a requirement of cells that display high concentrations of polyunsaturated fatty acids is an efficient antioxidant system for protection against peroxidative damage, which has been implicated in sperm dysfunction on numerous occasions (Jones et al., 1979; Froman and Thurston, 1981; Wishart, 1984; Alvarez and Storey, 1989; Selley et al., 1991; Cecil and Bakst, 1993; Hammerstedt, 1993); such reductions in antioxidant protection were observed with ageing in bulls, indicating increased susceptibility to peroxidative damage in older animals. Maximizing the potential in male animals requires a combination of optimal phospholipid fatty acid composition together with appropriate and adequate antioxidant protection. It is therefore of significance that in the present study the major changes in the phospholipid composition and associated reduction in amounts of polyunsaturation with ageing in bulls were accompanied by a marked reduction in the activities of the antioxidant enzymes glutathione peroxidase and superoxide dismutase within the seminal plasma.

The authors are grateful to the Scottish Office Agriculture and Fisheries Department for financial support, Newlands Cattle Breeding Centre, Scottish Livestock Services for their assistance and to the University of Glasgow for the William Stewart Scholarship for K. A. Kelso

\section{References}

Aitken RJ (1994) A free radical theory of male infertility Reproduction and Fertility Development 6 19-24

Alvarez JG and Storey BT (1989) Role of glutathione peroxidase in protecting mammalian spermatozoa from loss of motility caused by spontaneous lipid peroxidation Gamete Research 23 77-90

Bakst MR and Sexton TJ (1979) Fertilizing capacity and ultrastructure of fowl and turkey spermatozoa before and after freezing Journal of Reproduction and Fertility 55 1-7

Bearer EL and Friend OS (1982) Modifications of anionic-lipid domains preceding membrane fusion in guinea-pig spermatozoa Journal of Cell Biology 92 604-615

Brenner RR (1989) Factors influencing fatty acid chain elongation and desaturation. In The Role of Fats in Human Nutrition 2 nd Edn pp 46-79 Eds AJ Vergroesen and M Crawford. Academic Press, London

Cecil HC and Bakst MR (1993) In vitro lipid peroxidation of turkey spermatozoa Poultry Science 72 1370-1378

Christie WW (1982) Isolation of lipids from tissues. In Lipid Analysis; Isolation, Separation, Identification and Structural Analysis of Lipids 2nd Edn pp 17-25 Ed. WW Christie. Pergamon Press, Oxford

Christie WW (1988) Separation of molecular species of triacylglycerols by high-performance liquid chromatography with a silver-ion column Journal of Chromatography $454273-284$.

Christie WW, Noble RC and Moore JH (1970) Determination of lipid classes by gas chromatographic procedure Analyst $95940-944$ 
Darin-Bennett A, Poulos A and White IG (1974) The phospholipid and phospholipid-bound fatty acids and aldehydes of dog and fowl spermatozoa Journal of Reproduction and Fertility 41 471-474

Elias PM, Friend OS and Georbe J (1979) Membrane sterol heterogeneity: freeze fracture detection with saponin and filipin Journal of Histochemistry and Cyfochemistry 27 1247-1260

Evans RW and Setchell BP (1978) Association of exogenous phospholipid with spermatozoa Journal of Reproduction and Fertility 53 357-362

Froman DP and Thurston RJ (1981) Chicken and turkey spermatozoa superoxide dismutase: a comparative study Biology of Reproduction 24 193-200

Garner DL and Hafez ESE (1993) Spermatozoa and seminal plasma. In Reproduction in Farm Animals (6th Edn) pp 251-274 Ed. ESE Hafez. Lea and Febiger, Philadelphia

Griveau JF, Dumont E, Renard P, Callegari JP and Lannou D Le (1995) Reactive oxygen species, lipid peroxidation and enzymic defence systems in human spermatozoa Journal of Reproduction and Fertility 103 17-26

Hafez ESE (1993) Artificial insemination. In Reproduction in Farm Animals 6th Edn pp 424-440 Ed. ESE Hafez. Lea and Febiger, Philadelphia

Hammerstedt RH (1993) Maintenance of bioenergetic balance in sperm and prevention of lipid peroxidation: a review of the effect on design of storage preservation systems Reproduction and Fertility Development 5 675-690

Howarth B, Torregrossa D and Britton WM (1977) The phospholipid content of ejaculated fowl and turkey spermatozoa Poultry Science 56 1265-1268

Jain YC and Anand SR (1976) Fatty acids and fatty aldehydes of buffalo seminal plasma and sperm lipid Journal of Reproduction and Fertility 47 261-267

Jones R, Mann T and Sherins R (1979) Peroxidative breakdown of phospholipids in human spermatozoa, spermicidal properties of fatty acid peroxides and protective action of seminal plasma Fertility and Sterility 31 531-537

Kelso KA, Cerolini S, Noble RC, Sparks N and Speake BK (1996) The lipid and antioxidant changes in semen of the broiler fowl from 25 to 60 weeks of age Journal of Reproduction and Fertility 106 201-206

Lin DS, Connor WE, Wolf DP, Neuringer M and Hachey DL (1993) Unique lipids of primate spermatozoa desmosterol and DHA Journal of Lipid Research 34 491-499

Logue D and Greg A (1987). Infertility in the bull, ram and boar. Part 3. Collection and examination of semen In Practice 9 167-170

Mann T and Lutwak-Mann C (1981) Biochemistry of spermatozoa: chemical and functional correlations in ejaculated semen. In Male Reproductive Furction and Semen. Themes and Trends in Physiology and Biochemistry and Investigative Andrology pp 195-268 Eds T Mann and C Lutwak-Mann. Springer-Verlag, Berlin and New York; Lavenham Press Ltd., Suffolk
Neill AR and Masters CJ (1972) Metabolism of fatty acids by bovine spermatozoa Biochemistry Journal 127 375-385

Nissen HP and Kreysel HW (1983) Polyunsaturated fatty acids in relation to sperm motility Andrologia 15 264-269

Nissen HP, Kreysel HW and Schirren C (1981) Composition of the lipid-bound fatty acids of human semen in relation to its fertility values Andrologia 13 $444-451$

Oliw EH and Sprecher $\mathbf{H}$ (1989) Metabolism of polyunsaturated fatty acids by an (n-6) lipoxygenase associated with human ejaculates Biochemica et Biophysica Acta 1002 283-291

Olsen RE and Henderson RJ (1989) The rapid analysis of neutral and polar marine lipids using double-developed HPTLC and scanning densitometry Journal of Experimental Marine Biology and Ecology 129 189-197

Pickett BW, Komarek RJ (1966) Lipid and dry weight of bovine seminal plasma and spermatozoa from first and second ejaculates Journal of Dairy Science 30 742-746

Poulos A and White IG (1973) The phospholipid composition of human spermatozoa and seminal plasma Journal of Reproduction and Fertility 35 265-272

Poulos A, Darin-Bennett A and White IG (1973) The phospholipid-bound fatty acids and aldehydes of mammalian spermatozoa Comparative Biochemistry and Physiology 46B 54I-549

Ravie $\mathrm{O}$ and Lake PE (1985) The phospholipid bound fatty acids of fowl and turkey spermatozoa Animal Reproduction Science 9 189-192

Roldan ERS and Harrison RAP (1993) Diacylglycerol in the exocytosis of the mammalian sperm acrosome Biochemical Society Transactions 21 284-289

Scott TW (1973) Lipid metabolism of spermatozoa Journal of Reproduction and Fertility 18 65-76

Scott TW and Dawson MC (1968) Metabolism of phospholipids by spermatozoa and seminal plasma Biochemical Journal 108 457-475

Sebastian SM, Selvaraj S, Aruldhan MN and Govindarajulu P (1987) Pattern of neutral and phospholipids in the semen of normospermic, oligospermic and azoospermic men Journal of Reproduction and Fertility 79 373-378

Selley ML, Lacey MR, Bartlett CM and Ardlie NG (1991) Content of significant amounts of cytotoxic end-product of lipid peroxidation in human semen Journal of Reproduction and Fertility 92 291-298

Wishart GJ (1984) Effects of lipid peroxide formation in fowl semen on sperm motility, ATP content and fertilizing ability Journal of Reproduction and Fertility 71 113-118 